\title{
Qualitative reasoning in the education of deaf students: scientific education and acquisition of Portuguese as a second language
}

\author{
Heloisa Salles \\ Department of Linguistics, University of Brasilia \\ Brasília, DF, Brasil \\ hsalles@unb.br \\ Paulo Salles \\ Institute of Biological Sciences, University of Brasilia \\ Brasília, DF, Brasil \\ psalles@unb.br \\ Bert Bredeweg \\ Department of Social Sciences and Informatics, University of Amsterdam \\ Amsterdam, Netherlands \\ bert@swi.psy.uva.nl
}

\begin{abstract}
Brazilian educational system is faced with the task of promoting deaf people educational rights. Presently, the deaf are integrated in the classroom along with hearing students. Qualitative Reasoning may provide tools to support Portuguese acquisition in the context of the development of scientific concepts. This study describes an experiment with eight deaf students being exposed to three articulate qualitative models organized in gradual levels of complexity. Questionnaires were used to assess ther students' ability of expressing ideas in written Portuguese using the ontology provided by the models. An interesting result was that five students were consistent in the ability of recognizing objects and processes, build up causal chains and apply them to a given situation, assessing derivative values of quantities and making predictions about the consequences of changes, and write up a composition about an ecological accident, using linguistic descriptions of the relevant physical and social processes. These preliminary results are encouraging and ongoing work is the development of models and textual material in different domains, such as electrochemistry, to explore the potential of qualitative models in second language acquisition.
\end{abstract}

\section{Aspects of the linguistic and educational situation of the deaf in Brazil}

Two major problems arise in approaching the linguistic and educational situation of deaf people. Firstly, they have developed a natural sign language, which evolved over generations, enabling them to communicate and interact within their community. However, this situation is not as successful as it seems: there is great variation in the ability to use a sign language among the deaf, which in turn is due to a number of facts, such as age of exposure (if so) to sign language, family and educational support, the evolution of legal rights in the society etc. Secondly, due to the impairment in audio ability, it is very difficult for the deaf to use aural languages, which proves to be a strong barrier in their integration within the broad society, leading to their isolation and at best to their insertion in the so-called 'deaf culture'. As a corollary, a minority language, manifested in the visual modality, coexists with a dominant majority language, manifested in the aural modality.

As far as Brazilian deaf people are concerned, the situation is essentially as described above (cf. Ferreira Brito, 1993; Quadros, 1997; Salles et al. 2002). Given the cross-country heterogeneity in the educational system, we shall concentrate on a single situation, namely that of (few) deaf students in Brasília. Being the capital of the country, Brasília displays a wide system of state schools, which essentially assume the orientation in the 'Salamanca Declaration' regarding educational 'inclusion'. Accordingly, the deaf are integrated in the classroom along with hearing students. Portuguese being the official language in Brazil, there is a requirement on a bilingual education. In spite of all sorts of limitations, namely teachers qualification, financial support in the development and use of educational technology among others, most educational methods have been oriented by the assumption that Lingua Brasileira de Sinais (henceforth, LIBRAS), the 'Brazilian Sign Language', is the native language of the deaf community ${ }^{1}$, Portuguese being their second language. ${ }^{2}$ This policy has

${ }^{1}$ The Brazilian Federal Law $n^{0} 10.436$, published in 24/04/2002, legally recognizes LIBRAS as the language of the deaf community.

${ }^{2}$ Depending on educational orientation, some deaf students are worked as to develop aural abilities - also referred as lips reading. In the past, this procedure used methods prohibiting the use of a sign language, which is nowadays heavily criticized, given the understanding that the signed language is the most adequate for deafs, as a native language. Nowadays, it is still controversial that only the written version of the (second) aural language should be used for educational purposes. In the present study, the aural abilities of the student will not be taken into consideration, in spite of their (arguable) importance in the (written) use of the aural language. 
been responsible for the diffusion of LIBRAS within the educational community and among deaf students. Although the situation is far from ideal, various primary and secondary school teachers use LIBRAS or teach with the support of an educational interpreter/translator LIBRASPortuguese. Apart from this, some schools make use of special classrooms, exclusive for the deaf, which are intended to offer additional support with homework and written Portuguese. In this context, tools are required to articulate knowledge and to facilitate second language acquisition, in order to promote the social inclusion of the deaf.

Qualitative Reasoning (QR) is an area of AI that aims at the development of formalisms for reasoning with incomplete knowledge (B. Bredeweg and P. Struss (eds). 2003. Current Topics in Qualitative Reasoning. AI Magazine, special issue, Volume 24, Number 4, winter, pages 13-130).QR techniques are therefore taken to be powerful tools in the education of deaf students, given that they articulate knowledge about different physical and social systems in conceptual models; they use a restricted set of modelling primitives to represent a wide class of scientific concepts; they use a concise vocabulary, expressed in 'everyday language' to describe different classes of phenomena; they provide a clear description of the system structure; they have explicit representation of causal relations within the system, where it is possible to ground explanations about the system behavior. Due to the relevance of articulating different tools in the education, the importance of the present study is that it provides a formal representation of dynamic aspects of the systems under study in a situation involving students with special needs, to whom the use of logical reasoning embedded in a visual pedagogy in the development of their linguistic abilities proves to be of great interest, in a society seeking for human development.

Assumptions in this study are: (i) the (non-linguistic) diagrammatic representation of causal relations in qualitative models should be easily captured by deaf students due to their visual abilities (cf. Hawkins, 2001); (ii) the basic vocabulary should be familiar to them, due to their educational level (secondary school students); (iii) the vocabulary and the causal relations represented in the models should be understood, due to their ability to work out logical deductions; (iv) the understanding of the causal relations and the articulation of old and new vocabulary can be read off the linguistic description of physical and social processes and the textual connectivity in their written composition in Portuguese; (v) while conceptual connectivity (coherence) is a function of the understanding of the causal relations represented in the qualitative model, grammatical connectivity (cohesion) is a function of the level of proficiency in each language (cf. Halliday \& Hasan 1976; Koch, 2003, for details on the opposition coherence/ cohesion), LIBRAS, being expected to indicate native mastering, whereas Portuguese, some level of second language acquisition.
This paper is organized as follows: section 2 describes models and simulations developed for this experiment. Section 3 discusses methodological aspects of the experiment and the results are presented in section 4. Finally, discussion and final remarks are presented in section 5 .

\section{Models and simulations}

The Qualitative Process Theory (QPT) (Forbus, 1984) was chosen for this work because it provides an ontology for explicit representation of situations, processes and causal relations. Also, QPT was the basis for a number of studies in cognitive science. Of interest for this work are the theoretical framework for learning about physical domains defined by Forbus \& Gentner (1986) and the semantic studies of Kuhene \& Forbus (2002) and Kuhene (2003).

\section{The qualitative simulator}

The models were built in the modelling environment HOMER (Jellema, 2000; Bessa Machado \& Bredeweg, 2002), and simulations were run in the qualitative simulator GARP (Bredeweg, 1992). The visualizing tool VISIGARP (Bouwer \& Bredeweg 2001) was used to present the students diagrams representing objects and relations, quantities, quantity values, causal dependencies and state transitions during the simulations. Accordingly, the pair $<$ magnitude, derivative $>$ represented quantity values, and for the most important quantities quantity spaces were \{small, medium, large $\}$ and $\{$ hot, mild, cold $\}$. Causal relations are modelled by using two primitives: direct influences ( $\mathrm{I}+$ and $\mathrm{I}-$ ), used to represent the effects of processes, and qualitative proportionalities ( $\mathrm{P}+$ and $\mathrm{P}-$ ), used to represent how changes caused by processes propagate through the system.

\section{Model 1 - The 'growing tree'}

Deaf students were presented with three models. The first model represents a 'growing tree' and was used mainly for introducing the vocabulary and modelling primitives used to capture knowledge in the model. While a tree grows the area of its shade increases, which in turn causes soil temperature to decrease. Only process 'growth of the tree' is active. The model was used mainly for introducing the vocabulary and modelling primitives used to capture knowledge in a qualitative model. A simulation presented to the students showed 3 states after the initial scenario, with the directly influenced quantity biomass increasing from <small, increasing $>$ up to $<$ large, increasing $>$, while quantity shade changes through the same correspondent qualitative values, and the quantity temperature of the soil changes from $<$ hot, decreasing $>$ to $<$ cold, decreasing $>$.

\section{Model 2 - The 'drying shirt'}

This model describes changes in the weight of a shirt drying in the sun. The model explores a more complex situation, involving two processes (energy/heat production in the sun 
and evaporation of water in the shirt) and conceptually relates the shirt's weight with the mass of liquid water contained in it. A simulation presented to the students demonstrate that the directly influenced quantity liquid water mass changes from <large, decreasing $>$ to <small, decreasing $>$ and causes the quantity weight of the shirt to change through the same correspondent values, while the quantity vapour in the atmosphere was increasing.

\section{Model 3 - 'Cataguazes'}

This model is inspired in an ecological accident occurred in Cataguazes, a Brazilian city, involving the chemical pollution of several rivers in a densely populated area in the Paraíba do Sul river water basin, maybe the worse accident of this kind in the country (Martins, 2003). A paper industry used to store toxic chemicals it produces in a dam, managed by the industry itself. In March 2003, the dam broke down and 1.2 billion liters of pollutant substances were released and caused damages to water supply, soil, biodiversity and the economy in a large area of the water basin. Pollution went downstream and first contaminated the Pomba river, where fishing and cattle farms are important economic activities. As the mass of pollutants kept flowing downwards, it reached the Paraíba do Sul river, where half million people was affected. Companies that explore the socalled 'freshwater shrimp' in that area reported heavy economic losses, and jobs were lost. The model includes notions of management and removal of pollutants from the dam to demonstrate the effects of good and bad management.
Vocabulary, cause - effect relations and logical deductions were explored in the experiment by using the Cataguazes model. Its causal model was also the basis for a written exercise (see Section below). Objects, quantities and causal relations of the Cataguazes model are presented in Figure 1.

\section{A simulation with the 'Cataguazes' model}

We present here, to illustrate, a simulation with the Cataguazes model. The initial scenario describes a bad situation: quantity pollution has value $<$ large, increasing $>$ at the dam and the rivers, while cattle and shrimp have values $<$ small, decreasing $>$ and jobs in both activities are also $<$ small, decreasing $>$. The simulation shows the effects of greater control on the pollution that leads to an increasing amount of pollutants being removed from the dam. A behaviour path $[1 \rightarrow 2 \rightarrow 3 \rightarrow 10 \rightarrow 11 \rightarrow 14 \rightarrow 12 \rightarrow 22$ $\rightarrow 20$ ] was selected to be presented to the students because it shows the ideal results: in the states 1, 2, and 3 the management effects cause the reversion in the derivatives of the instances of pollution. Next, this quantity decreases first in the dam (states 3,10,11), then in the Pomba river (states $11,14,12$ ) and finally the conditions improve in the Paraíba do Sul river (states 12, 22, 20). Economic activities follow improvement in river water quality. These results are shown in Figures 2 and 3.

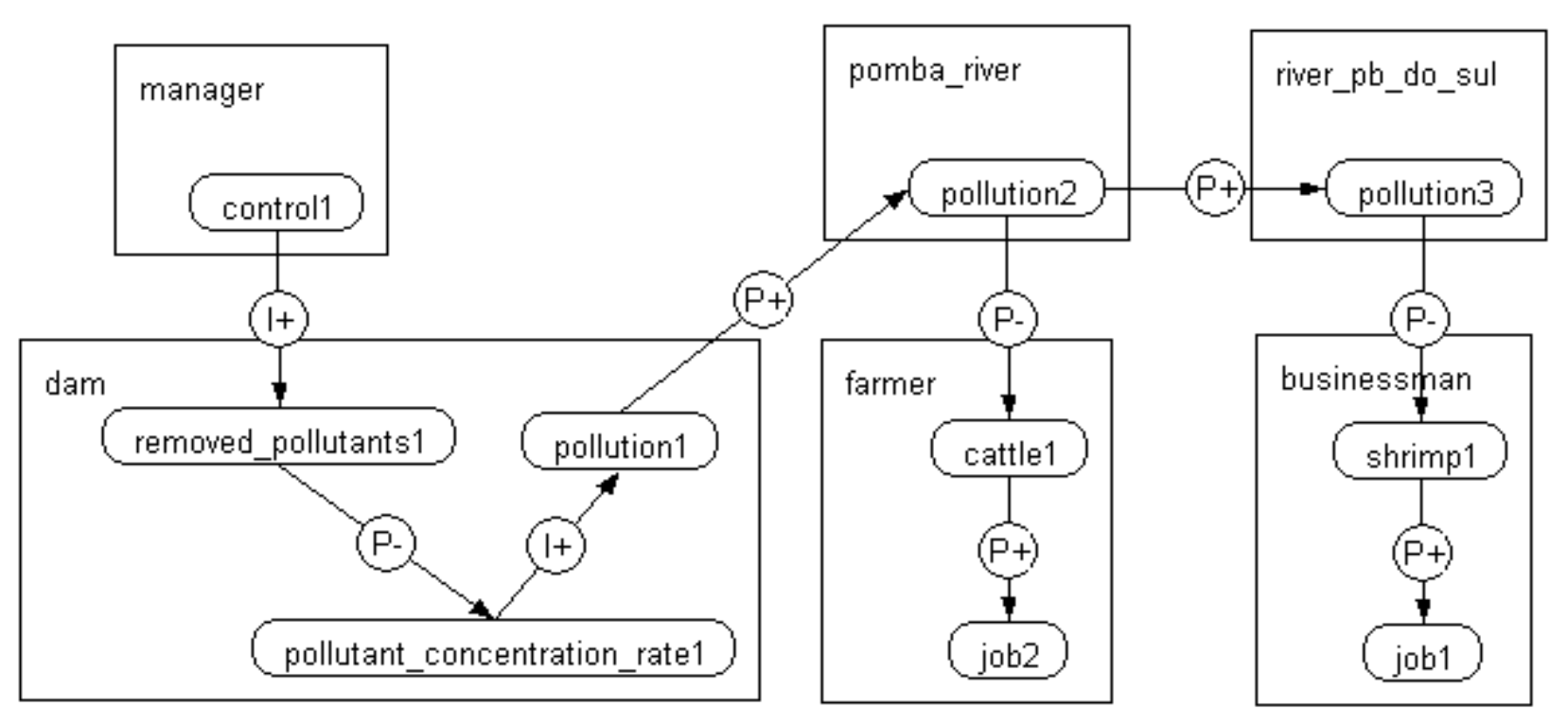

Figure 1: Objects manager, dam, farmer, businessman, Pomba river and Paraíba do Sul river, associated with quantities and causal relations in the Cataguazes model. 


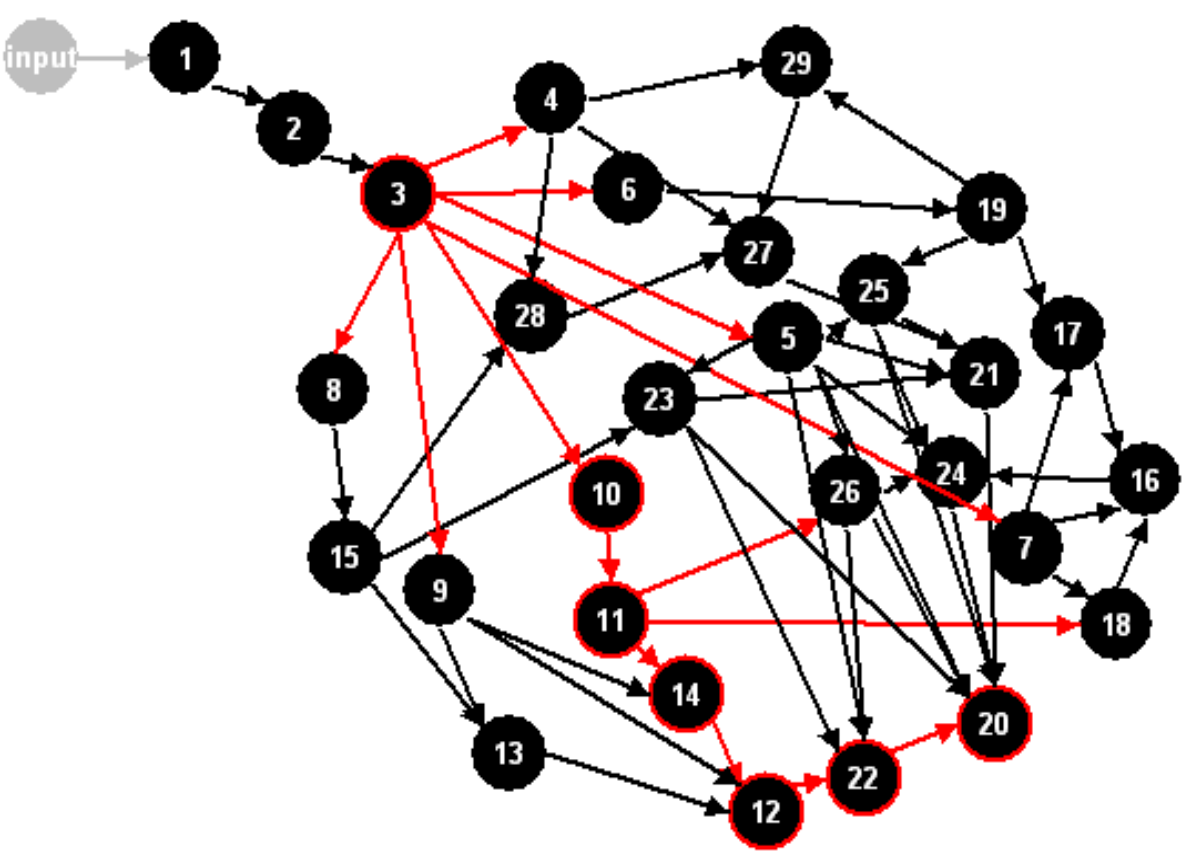

Figure 2. State-graph of a simulation on good management of the Cataguazes model

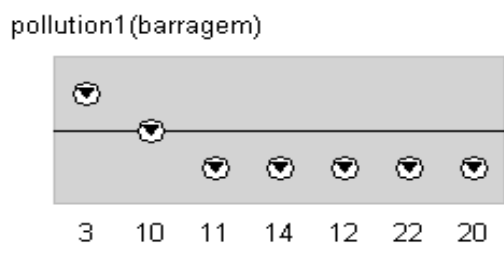

pollution3(rio_pomba)

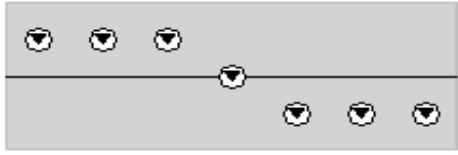

$\begin{array}{lllllll}3 & 10 & 11 & 14 & 12 & 22 & 20\end{array}$

pollution2(rio_pb_do_sul)

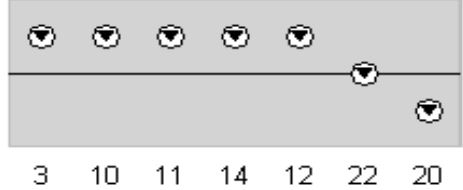

\section{grande \\ medio \\ pequeno}

grande

medio

pequeno

grande

medio

pequeno

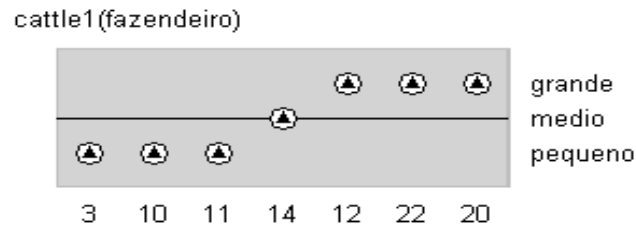

job2(fazendeiro)

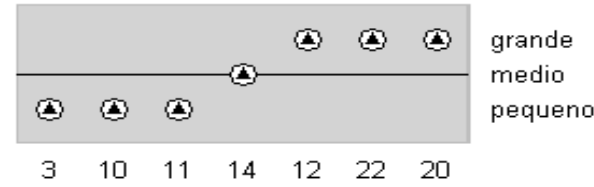

snrımpi(empresarı)

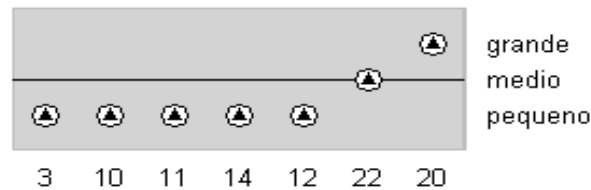

job1 (empresario)

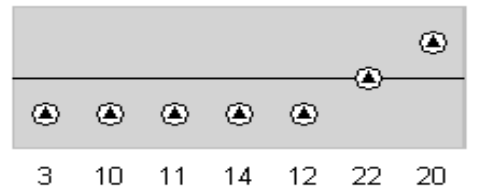

grande medio pequeno

Figure 3. A selected simulation of good management using the 'Cataguazes' model. (a) values of concentrations of pollution in the dam and in two rivers; (b) recovering of cattle and farming jobs in the Pomba river area; (c) recovering of shrimp and related jobs in the Paraíba do Sul 


\section{Methodology}

\section{Subjects}

This study is a joint activity in a secondary state school, with deaf students from the $2^{\text {nd }}$ year and their teachers and interpreters of LIBRAS-Portuguese in the classroom. The students are fluent in LIBRAS and display some knowledge of Portuguese as a second language, given their exposure to this (written) language since their early (formal) education. The students were exposed to three qualitative models organized in gradual levels of complexity. As for their background, they are familiar with some of the scientific concepts articulated in the models, due to their education in physics, chemistry and biology, although unfamiliar with their expression in modelling environments. ${ }^{3}$

\section{The goals}

We are not measuring learning. The goal of the present study is to verify the understanding of the deaf students of causal relations expressed in the models by means of the following instruments: (i) the manipulation of logical implications within the models; (ii) the linguistic description of physical and social processes referring to the causal models and to analogous situations, using LIBRAS and (written) Portuguese; (iii) the use old and new vocabulary in LIBRAS and (written) Portuguese.

\section{How to present the models}

The methodology used in this experiment draws on concepts developed in Forbus \& Gentner (1986) and in Salles et al. (2003). From the first work, we used an approach based on the sequence from perceptual-based representations acquired earlier in the learning process towards sparse and abstracts representations of the domain. We informally followed a canonical learning sequence that corresponds to (i) diagrammatic and verbal everyday problems (protohistories); Next, (ii) the students were stimulated to find out causality in their working models. At that point, (iii) typical representations of qualitative reasoning became insightful for the students ("naive physics"). This experiment did not go for the last step, (iv) the development of expert models (Forbus \& Gentner, 1986).

From Salles et al. (2003) we adopted a curriculum based on the idea of model progression along different dimensions, exploring knowledge spanning from simple to complex problems, and from local to wide area of coverage. Model progression based on structural changes was adopted at a general level in the experiment. So the sequence ('growing tree' model $\rightarrow$ 'drying shirt' model $\rightarrow$ 'Cataguazes' model)

\footnotetext{
${ }^{3}$ In spite of the above-mentioned heterogeneity in the use of sign languages by the deaf, we shall assume these students to be proficient in LIBRAS - a detailed analysis of their linguistic abilities in LIBRAS would take us too far a field - being a topic for future research. A superficial inquiry has shown that they have been exposed to LIBRAS for at least 8 years in school.
}

included models representing the effects of one process, two processes with few objects and quantities, and two processes with many objects and quantities. Some features of the models could be explored according to some dimensions (generalization / specialization, analogy, inverse, structural changes, orders). Analogies were used in a number of cases, as for example, in comparing the effects of cattle and shrimp mortality on the availability of jobs.

\section{The sessions with the students}

The experiment was run in three sessions, one for each model. During the sessions the number of students changed. Only three (students 1,2,3) have gone through all the 3 models. Four students explored models 2 and 3, and one student only the third model. Eventually these eight students all completed the last task (to write the essay). An educational interpreter/translator LIBRAS-Portuguese was present throughout the experiment. The most relevant vocabulary was written in Portuguese in the blackboard and was pointed out several times during the activity. We used a projector to show the models and run the simulations to the students in real time. Explanations given by the teacher were interpreted / translated in LIBRAS. After the presentations, details of structure and behavior of the systems were discussed, until the students said they had 'understood' the models. At this point, we started the evaluation process.

\section{Evaluation of the understanding of the models}

The tools used for the evaluation of the experiment were questionnaires and VISIGARP screenshots to illustrate the questions. Most of times, VISIGARP's options 'E/R structure' (for objects and relations) and 'Dependencies' (for the causal model) were selected. The students were tested in (written) Portuguese on the understanding (i) of representational aspects, such as quantity, objects (by answering questions); (ii) of causal model diagrams (by identifying positive and negative influences, drawing arrows and answering questions); (iii) of if-then implications regarding cause-effect relationships (by filling in blank spaces in sentences); (iv) of providing explanations by backtracking the causal model (by filling in blank spaces in sentences); and (v) by writing an essay about a topic presented in a qualitative simulation model. The questionnaire consisted of 12 questions (Q1-Q12) distributed among the three models. The questions are explained below.

\section{Questions about the 'growing tree' model}

Five questions were presented to the students about the 'growing tree' model, with the following objectives: a) to identify the names of two objects in a list (Q1); b) to identify a process and to assess two utterances about influences on quantities (Q2); c) to count states in a state graph (Q3); d) and to identify the two values - magnitude and derivative - of a quantity represented in a diagram: 
what is the value of quantity $X$ in state 2?; is this quantity increasing or decreasing? (Q4 and Q5).

\section{Questions about the 'drying shirt' model}

Three questions explored the 'drying shirt' model, with the following objectives: a) in a set of 4 names of things, identify 2 objects and 2 quantities (Q6); b) to recognize the two active processes in a particular state $(\mathrm{Q} 7)$; c) to fill in the blanks spelling out 5 consequences of a particular change in the system obtained by means of logical deduction: IF quantity $X$ is increasing, THEN quantity $Y$ is decreasing, quantity $Z$ is increasing, and quantity $W$ is decreasing (Q8).

\section{Questions about the 'Cataguazes' model}

The 'Cataguazes' model was explored by four questions, with the following objectives: a) to draw a causal diagram of the accident using GARP's modelling primitives to complete a causal model in which 6 proportionalities were missing (Q9); b) to spell out the consequences of a particular change in the system, in which 4 predictions (expressed as if-then utterances) should be made (Q10); c) to explain the behavior of a particular quantity making references to 4 previous steps, by means of backtracking the causal model: quantity $W$ is decreasing BECAUSE quantity $Z$ is increasing, (...) until, at the end of the chain, the process $P$ caused quantity $X$ to increase) (Q11). The final question (Q12) was a written assignment.

\section{The results}

Eight students wrote essays for Q12. Five of them produced good texts and their work was analyzed here. The results of the remaining three students were not conclusive and were not included in the following discussion.

\section{Answers about the 'growing tree' model}

Three students answered the questions referring to model 1. Initially they were not able to identify the objects:

$(\mathrm{Q} 1=[1 / 2 ; 1 / 2 ; 0 / 2])^{4}$. Also it was not easy to identify the process: $(\mathrm{Q} 2=[0 / 1 ; 0 / 1 ; 1 / 1])$, but they could assess utterances about the influences of quantities: $(\mathrm{Q} 2=[1 / 2 ; 2 / 2$; $2 / 2]$ ). All of them were able to count the number of states in the state graph $(\mathrm{Q} 3=[1 / 1 ; 1 / 1 ; 1 / 1])$. At this point, the students were not so good at identifying magnitudes $(\mathrm{Q} 4=[1 / 2 ; 1 / 2 ; 0 / 2])$ and derivatives $(\mathrm{Q} 5=[0 / 2 ; 2 / 2 ; 1 / 2])$ of selected quantities.

\footnotetext{
${ }^{4}$ Some answers given by the students are presented as ratios (e.g. $2 / 3$ ), in which the number above (2) represents the number of correct answers given by the student, and the number below (3) the total of answers the student should provide in that question. The results of the group are presented between brackets. For example, the answers of the students 1,2 , and 3 are, respectively, [0/2;2/2; $1 / 2]$. Note that the ordered presentation of each student's results is kept throughout the paper, so that the reader can follow the performance of each student, until the results of student 5 in the 'Cataguazes' model.
}

\section{Answers about the 'drying shirt' model}

Answering questions about the 'drying shirt' model, the students now could identify the objects $(\mathrm{Q} 6=[1 / 3 ; 3 / 3 ; 3 / 3$; $3 / 3]$ ) and the quantities (Q6=[4/6;3/6;6/6;4/6]). Three out of four students were able to identify the processes: $(\mathrm{Q} 7=[2 / 2 ; 2 / 2 ; 0 / 2 ; 2 / 2])$. Important to note that the student who did not identify the processes demonstrate to be aware of the connection between 'process', 'rate' and the 'I' symbol. Finally, half of the students were not able at this stage to make predictions about the behavior of quantities: $(\mathrm{Q} 8=[0 / 5 ; 4 / 5 ; 3 / 5 ; 0 / 5])$.

\section{Answers about the 'Cataguazes' model}

With respect to the 'Cataguazes' model, the students were not able to draw the causal chain by themselves $(Q 9=[0 / 6$; $4 / 6 ; 2 / 6 ; 0 / 6 ; 0 / 6])$. However, giving them the causal model, they were good in making predictions: $(\mathrm{Q} 10=[3 / 4 ; 4 / 4 ; 3 / 4$; $4 / 4 ; 4 / 4])$. Two students were not able to move backward between the nodes of the causal model to build explanations: $(\mathrm{Q} 11=[0 / 4 ; 4 / 4 ; 4 / 4 ; 4 / 4 ; 0 / 4])$.

\section{The written composition}

The final task was to write up an essay based on the causal model of 'Cataguazes'. All the students produced a text pretending they were going to present on TV a report of the accident. Our evaluation consisted of counting the number of occurrences of typical expressions of cause - effect relations, such as when the pollution in river $P$ increases, the cattle dies, and combine it with the size of the text. For example, 5/20 means five occurrences in 20 lines. The results are $(\mathrm{Q} 12=[5 / 20 ; 5 / 21 ; 7 / 11 ; 7 / 7 ; 4 / 7])$. Although interesting to show that few students wrote very predictive texts (that is, many predictions in few lines, for example, $7 / 7,7 / 11$ ), these results do not necessarily express the richness of the texts. We leave for future work to further explore these essays.

\section{Some problems the students found}

The performance of this group of five students allows for interesting observations and conclusions. Notorious was the evolution of their skills along the experiment. In the model 3 , most of them were able to play with the causal model and do forward (predictive) and backward (explicative) reasoning over the causal model. However, the analyses discussed here do not capture some subtle details. For example, most of the students were able to recognize wellknown processes, such as evaporation, but it was not clear for them how such processes work, and why pollution control in the dam or energy generation by the sun can be also seen as processes.

They also had problems with the causal models, especially with long causal chains, involving three or more quantities. For example a student can easily say that if $X$ is increasing, then $Y$ is decreasing from the causal model but still find it difficult to infer what happens to $Z$ if the reasoning requires the computation of inferences such as $[X$ influences $Y$ and $Y$ 
influences $Z$ ]. Another difficult aspect for the deaf was the use of incomplete causal models. For example, in Q9 the students received the causal model of 'Cataguazes' and they should fill out the 6 missing proportionalities. The students scored very low in this question.

A similar problem happened in questions Q10 and Q11. In both cases, one of the initial or intermediate values was given, and the student was expected to continue from that point. Some students ignored this given value and built their own predictions (correctly). For example, in Q11 there were some blanks to be filled: At a certain point, jobs with shrimp because the quantity of shrimp _ the pollution in the Paraiba do Sul river _ DECREASED_ (...). We expected the answers \{increased, increased\}. However one student wrote \{decreased, decreased\}, changed over the situation in the Paraíba do Sul river to INCREASED pollution, and continued creating a complete and correct sequence of influences. Obviously, we accepted the answer as correct.

\section{Discussion and final remarks}

This paper describes a preliminary study on the use of qualitative models to support second language acquisition by deaf students in the context of science education. After being exposed to three qualitative models of increasing complexity, the students answered a set of questions about the concepts expressed in the models. Having considered quantitative and qualitative analysis of the questionnaires, we can say the students were: $\underline{\text { Successful in recognizing }}$ objects and their quantities; Successful in identifying changes in the values of quantities (magnitudes and derivatives) during simulations; Successful in building up causal chains based on the given models; Partially successful in identifying processes often approaching them from the representation of their rate (for instance: evaporation rate); Partially successful in building up causal chains following initial values assumed for some quantities in the question; Mostly successful in making use of the causal relations for explaining and discussing facts of Cataguazes in the (written) composition; Successful in reporting consequences of the Cataguazes ecological accident in a (written) composition based on the causal relations represented in the model.

Although this experiment presents an initial overview of the possible use of qualitative models in science education to support second language acquisition, the consistence in the results allows for a correlation between the writing skills of the students and their understanding of the causal model. In particular, conceptual connectivity in the text seems to be a function of the ability to recognize objects and processes, to build causal chains and to apply them to a given situation, assessing derivative values of quantities and making predictions about the consequences of their changes. In fact, while being exposed to causal models, these students wrote up their composition about the ecological accident in Cataguazes, making use of coherent reasoning, which can be inferred from the linguistic descriptions of the physical and social processes. This is an interesting result, given the well-known difficulties faced by deaf students in this task which in a way extends to all students in the educational environment.

The results reported in this paper constitute a preliminary approach in a research program concerned with the acquisition of Portuguese as second language by deaf students having science education as a background. The research program provides the theoretical basis for establishing different levels of proficiency in Portuguese of deaf students, and for the development of educational tools, such as bilingual dictionaries of specialized language, methods and techniques in (second) language teaching and procedures in translation / interpretation in the scientific education. Lessons learned in the experiment described here are useful for the design of interactions with the deaf involving knowledge communication supported by qualitative models. As part of these efforts, a similar experiment is being prepared to explore a qualitative model of the Daniell cell, developed to enhance the understanding of electrochemistry in secondary schools (Salles et al. 2004).

\section{Acknowledgments}

We thank the deaf students that took part in the experiment, as well as their teachers and educational coordinators. Thanks also to the APADA for their support. H. Salles and P. Salles are grateful to CAPES/MEC/ PROESP for the grant supporting the research project "Português como segunda língua na educação científica de surdos" ('Portuguese as a second language in the scientific education of the deaf').

\section{References}

Bessa Machado, V. \& Bredeweg, B. (2002) Investigating the Model Building Process with HOMER. In Bredeweg, B. (Editor) Proceedings of the International workshop on Model-based Systems and Qualitative Reasoning for Intelligent Tutoring Systems, pages 1-13, San Sebastian, Spain, June $2^{\text {nd }}, 2002$.

Bouwer, A. and Bredeweg, B. (2001) VISIGARP: Graphical representation of qualitative simulation models. In Moore, J.D.; Luckhardt Redfield, G. \& Johnson, J.L. (Editors) Artificial Intelligence in Education: AI-ED in the Wired and Wireless Future. Amsterdam, IOS Press, pp. 294-305.

Bredeweg, B. (1992) Expertise in Qualitative Prediction of Behaviour. Ph.D. thesis, University of Amsterdam, Amsterdam, The Netherlands, 1992.

Ferreira Brito, L. 1993 Interação Social e Integração de Surdos. Rio de Janeiro, Babel.

Forbus, K.D. (1984) Qualitative process theory. Artificial Intelligence, 24:85-168.

Forbus, K. \& Gentner, D. (1986) Learning physical domains: toward a theoretical framework. In Michalsky, R.S.; Carbonell, J. \& Mitchell, T. (eds.) Machine Learning: an Artificial Intelligence Approach. vol. 2, Los Altos, CA, Morgan Kaufman Pub., 311-348. 
Hawkins, R (2001) Second Language Syntax. London, Blackwell.

Halliday, M. A. K. \& Hasan, R. (1976) Cohesion in Spoken and Written English. London, Longman.

Jellema, J. (2000) Ontwerpen voor ondersteuning - De rol van taakkennis bij ondersteuningsontwerp. Master thesis, University of Amsterdam, Amsterdam (in Dutch).

Koch, I. (2003) Desvendando os Segredos do Texto. São Paulo, Cortez.

Kuhene, S. \& Forbus, K. (2002) Qualitative physics as a component in natural language semantics: a preliminary report. In Agell, N. \& Ortega, J.A. (eds.) Proceedings of the Sixteenth International Workshop on Qualitative Reasoning (QR'02), pages 91-97 Sitges - Barcelona, Spain, June 10-12, 2002.

Kuhene, S. (2003) On the representation of physical quantities in natural language. In Salles, P. \& Bredeweg, B. (eds.) Proceedings of the Seventeenth International Workshop on Qualitative Reasoning (QR'03), pages 131138 Brasília, Brazil, August 20-22, 2003.

Martins, J. (2003) Uma onda de irresponsabilidades. Ciência Hoje, 33(195): 52-54.

Quadros, R. (1997) Educação de Surdos: a Aquisição da Linguagem. Porto Alegre, Artes Médicas.

Salles, H. M. L.; Faulstich, E.; Carvalho, O.L. \& Ramos, A.A.L. (2002) Ensino de Língua Portuguesa para Surdos - Caminhos para a Prática Pedagógica. Secretaria de Educação Especial. MEC, Brasil.

Salles, P. \& Bredeweg, B. (2003) A case study of collaborative modelling: building qualitative models in ecology. In Hoppe, U.; Verdejo, F. \& Kay, J. (eds.) Artificial Intelligence and Education: shaping the future of learning through intelligent technologies. Amsterdam, IOS Press / Omasha, pp. 245-252.

Salles, P.; Gauche, R. \& Virmond, P. (2004) A qualitative model of the Daniell cell for chemical education. Proceedings of the $18^{\text {th }}$ International Workshop on Qualitative Reasoning (this volume). 